\title{
ESTADO DE BIENESTAR Y TURISMO PARA TODOS. MOTIVOS PARA NO VIAJAR
}

\author{
Antonio Alvarez-Sousa
}

Universidade da Coruña

\section{RESUMEN}

El objetivo de esta investigación es analizar las características de las personas que no realizan viaje turístico en la Unión Europea y los motivos que le llevan a ello. Nos basamos en las desigualdades sociales analizadas a partir de variables individuales y contextuales. También estamos interesados en conocer cómo influyen las situaciones de crisis que afectaron a ciertos países de Europa. Empleamos los datos del Eurobarómetro. Aplicamos la técnica de Generalized Structural Equation Modeling (GSEM). Los resultados son que el no realizar viaje turístico se debe fundamentalmente a razones económicas y de tiempo libre que están influenciados por el modelo de Estado de Bienestar, las condiciones sociales de existencia y la percepción de riesgo de la crisis.

Palabras clave: teoría crítica; justicia social; Estado de Bienestar; estratificación social; crisis; turismo para todos; Generalized Structural Equation Model (GSEM).

\section{The welfare state and tourism for all.}

Reasons why people do not travel

\section{ABSTRACT}

The aim of this research is to analyse the characteristics of the people that do not travel for tourism purposes in the European Union and the reasons behind their decision. Social inequalities form the starting point of our research, based on a series of individual and contextual variables. A further issue for consideration is the influence exerted by the economic crises that affected a number of European states. We used Eurobarometer data

Recibido: 20 de septiembre de 2017

Devuelto para su revisión: 15 de diciembre de 2017

Aceptado: 30 de enero de 2017

Departamento de Sociología y Ciencias de la Comunicación. Facultad de Sociología. Universidade da Coruña. Campus de Elviña. 15071 A CORUÑA (España).E-mail: sousa@udc.es 
and applied Generalized Structural Equation Modeling (GSEM). The results show that the decision not to travel for tourism purposes is attributable mainly to economic and free time causes, influenced by the welfare state model, social living conditions and the perception of the risks associated with situations of economic crisis.

Keywords: critical theory; social justice; welfare state; social stratification; crisis; Tourism for all; Generalized Structural Equation Modeling (GSEM).

\section{INTRODUCCIÓN}

Existe un importante porcentaje de ciudadanos de la Unión Europea que no pueden realizar viaje turístico. Según los datos del eurobarometro 414, en el año 2014 el $28.05 \%$ del total de los entrevistados no realizaron viaje. Si ponderamos los datos para el total de la Unión Europea resulta que no realizaron viaje el $25.78 \%$ de los ciudadanos.

Esta investigación se enmarca dentro de lo que Tribe (2010) consideró la perspectiva crítica en turismo y más precisamente dentro de lo que Cohen and Cohen (2012) denominaron el área de conocimiento de la "justicia social y el turismo", el cual parte de la idea compartida con Pritchard et al. (2011) de que no es suficiente con un conocimiento instrumental sobre el turismo, sino que es necesario que los académicos participen en la creación de un mundo más sustentable y en esa línea se incluye el análisis de los grupos sociales más desfavorecidos del sistema que tienen problemas para realizar viaje. El turismo antes que un lujo debe de ser un derecho humano. Para solucionar el problema se suele proponer el turismo social como una forma de distribución equitativa (McCabe, 2009; Minneart et al., 2009; Casey, 2010), pero además del turismo social es necesario solucionar los problemas que llevan a no poder viajar.

El objetivo general de la presente investigación es analizar las desigualdades en el acceso al viaje turístico y los motivos de los ciudadanos de la Unión Europea para no realizan viaje turístico. Sólo conociendo los motivos y asociándolos con los distintos grupos sociales y contextos se pueden proponer soluciones al problema. Las preguntas y los objetivos concretos de investigación son por lo tanto: ¿cuáles son los motivos por los que algunas personas no pueden realizar viaje turístico?, ¿cuáles son las características de los grupos sociales que no pueden realizar viaje turístico?, ¿existen diferencias según el modelo de Estado de Bienestar de los países donde viven?, ¿afectó la crisis económica a la realización de viaje turístico por sus ciudadanos?.

\section{FUNDAMENTOS TEÓRICOS}

En los estudios de turismo no podemos olvidar la perspectiva crítica de la sociedad (Tribe, 2010), la cual tiene implicaciones ontológicas (Atelevic et al., 2005) que hace avanzar las políticas hacia una sociedad más equitativa en la que el bienestar social se extienda a todas las personas, es lo que Cohen y Cohen (2012) denominan la perspectiva de la justicia social y el turismo. 


\subsection{El turismo como un derecho: turismo para todos}

En la perspectiva del turismo como un derecho destacan los análisis del turismo social y la justicia social que ha de entenderse en un doble sentido, haciendo referencia por una parte al "Tourism for All" y por otra parte al "Solidarity Tourism" (Organisation Iternational du Tourisme Sociale, 2018). En esta investigación nos dedicaremos a analizar la primera de ellas, cómo incrementar la participación en el turismo de los grupos desaventajados.

El turismo ha de considerarse como un derecho para todas las personas (Hall and Brown, 2012) en una "welfare society". Las instituciones han de conocer los motivos que tienen las personas que no pueden realizar viaje turístico para poder remediarlos. Esta idea la fundamentamos en las distintas resoluciones de Naciones Unidas como son la Declaración Universal de los Derechos Humanos -artícule 24: “Todas las personas tienen derecho al descanso y el ocio, incluyendo una limitación razonable de las horas de trabajo y un período de vacaciones pagadas" (United Nations, 1948)-; Pacto Internacional de Derechos Económicos, Sociales y Culturales -artículo 7d: "Los Estados pertenecientes al Pacto reconocen los derechos e todo los ciudadanos al disfrute de condiciones de trabajo equitativas y satisfactorias que garanticen, en particular: descanso, ocio y limitación razonable de las horas de trabajo y vacaciones periódicas pagadas, así como la remuneración de los días festivos" (United Nations, 1966)-; Código Ético Mundial para el Turismo -artículo 7: "Derecho al Turismo" (United Nations, 2001)-. El derecho al turismo para todos también está recogido en el Dictamen sobre el Turismo Social en Europa, del Comité Économique et Social Européen (2006) que en su artículo 2.1 dice que toda persona tiene derecho al descanso y al ocio, siendo el derecho al turismo una concreción de ese derecho general.

\subsection{Factores que condicionan los motivos para no realizar viaje turístico}

Aunque hay autores que sostienen la homogeneización del consumo de ocio, con la eliminación de las diferencias de status por la desaparición de las clases (Lipovetsky, 2004, 2006), desde la perspectiva de las teorías críticas (Bourdieu, 1979; Bertoncelo, 2013; Chan \& Goldthorpe, 2007; Warnier, 1999; Wuggenig, 2007) se sostiene que sigue existiendo diferencias en consumo entre los distintos estratos sociales y en concreto para el consumo turístico (Ying et al., 2016). La falta de recursos económicos y de tiempo libre que impiden realizar viaje turístico no afectan a todos los grupos sociales por igual ni a las personas que viven en los distintos modelos de Estado de Bienestar, ni se ven afectados del mismo modo por las crisis.

Existen diferencias entre los distintos estratos sociales según las condiciones sociales de existencia. Para analizar la estratificación social y las diferencias de consumo de ocio no es suficiente con una perspectiva unidimensional, sino que es necesario tener en cuenta distintas variables que Goldthorpe (2013) y Bourdieu (1979) asocian con la ocupación, los estudios, el género, la edad y el hábitat. Los distintos estratos tienen diferentes recompensas económicas y de tiempo libre (Davis y Moore, 1970), sean merecidas (conformistas) o inmerecidas (críticos), pero el caso es que las tienen y además las emplean como símbolos de distinción (Bourdieu, 1979). 
Además de la estratificación social, es necesario diferenciar el contexto donde se vive, que está marcado por los distintos de modelos de Estado de Bienestar (Domingo y Morales, 2016). Aunque algunos autores consideran que se tiende hacia una convergencia en Europa (Schmitt, 2011; Paetzold, 2013), nosotros partimos de que siguen existiendo grandes diferencias que se ven reflejadas en el acceso al consumo de ocio y turismo, con comportamientos heterogéneos (Eugenio-Martin y Campos-Soria, 2014). Para diferenciar los modelos de Estado de Bienestar tomamos como referencia la teoría de Esping-Andersen (1990, 1999, 2000a, 2000b) y algunos de sus seguidores (Hemerijck, 2000), con los añadidos posteriores que se hicieron del modelo Mediterráneo (Moreno, 2001) y en la actualidad de los países de la Europa del Este (Deacon, 2000; Kouba \& Grochová, 2013). Diferenciaremos así entre el modelo Nórdico, el Continental, el Liberal, el Meridional y del Este.

Entro los modelos clásicos -sin incluir el del Este- existe diferencias relacionadas con la equidad y la eficiencia (Sapir, 2006). El que reúne las dos características en grado sumo es el Nórdico. El Meridional es el considerado menos eficiente y con más baja equidad. El Liberal se considera que tiene elevada eficiencia pero baja equidad y el Continental que tiene baja eficiencia pero elevada equidad. La principal característica del modelo Meridional es que las familias siguen siendo las responsables del bienestar de los ciudadanos, siendo las mujeres las que se ocupan en mayor medida de proporcionar los servicios personales de forma no mercantilizada y los hombres tienen mayor papel en la aportación de ingresos (Flaquer, 2004). Estas características han de verse reflejadas en la realización o no de viaje y en los motivos de no realización de viaje turístico. Los que habitan en países del modelo Nórdico se espera que tengan menos problemas y los que habitan en países del modelo Meridional y del Este que tengan más problemas.

Respecto a la comparación entre los modelos del Este y al Meridional, en principio puede suponerse que está más desarrollado el bienestar en el modelo Meridional, pero en la actualidad con la crisis que está afectando a los países Meridionales, es posible que incluso superen en problemas para no realizar viaje turístico a los de la Europa del Este. Si recurrimos a los datos sobre la situación económica de los hogares -medido por las dificultades para pagar facturas a fin de mes-, la satisfacción con la vida, la visión de cómo está afectando la crisis, podemos ver cómo en pleno momento de la crisis los que tienen más problemas en estos momentos son los del modelo Meridional, incluso por encima del de la Europa del Este (ver tabla 1). Esto ha de verse reflejado en la realización del viaje turístico.

La crisis económica que afectó a ciertos países de la UE a partir del año 2007 es percibida como un riesgo (Beck, 1998) que afecta al consumo en ocio y en turismo. Además, la crisis puede estar afectando al incremento de las diferencias entre los grupos sociales. Semeeding and Thompson (2011) consideran que los hogares de mayor renta no están afectados por las crisis como los hogares con baja renta, y si les afecta, pronto recuperan niveles anteriores. Las crisis económicas están relacionadas con descensos de consumo, además de cambios en la estructura del consumo, centrándose más en satisfacción de necesidades básicas frente a consumos superfluos o conspicuos, lo que se puede llamar consumo defensivo (Brändle, 2010). Las distintas zonas de la Unión Europea se vieron afectadas de distinto modo por la crisis que conllevó comportamientos heterogéneos ante el viaje turístico (Eugenio-Martin y Campos-Soria, 2014). 


\section{Tabla 1}

POBLACIÓN QUE MUESTRA DIFICULTADES PARA PAGAR FACTURAS, NO SATISFECHOS CON LA VIDA Y QUE CONSIDERAN QUE SU VIDA Y LA DEL ÁREA DONDE HABITAN EMPEORARON EN LOS ÚLTIMOS CINCO AÑOS, SEGÚN LOS DISTINTOS MODELOS DE ESTADO DE BIENESTAR (\%)

\begin{tabular}{|l|r|r|r|r|}
\hline $\begin{array}{c}\text { Modelo } \\
\text { Estado } \\
\text { Bienestar }\end{array}$ & $\begin{array}{c}\text { Dificultades para } \\
\text { pagar facturas a fin } \\
\text { de mes, la mayor } \\
\text { parte de las veces }\end{array}$ & $\begin{array}{c}\text { No } \\
\text { satisfecho } \\
\text { con la vida }\end{array}$ & $\begin{array}{c}\text { Su vida fue a } \\
\text { peor en los 5 } \\
\text { últimos años }\end{array}$ & $\begin{array}{c}\text { El área donde vive } \\
\text { ha empeorado en los } \\
\text { últimos cinco años }\end{array}$ \\
\hline Nórdico & 2.86 & 3.8 & 17.41 & 10.1 \\
\hline Continental & 7.3 & 12.92 & 26.24 & 12.66 \\
\hline Liberal & 11.75 & 9.83 & 31.51 & 46.19 \\
\hline Meridional & 23.09 & 40.5 & 59.72 & 25.12 \\
\hline Este & 14.89 & 37.59 & 46.78 & 14.53 \\
\hline
\end{tabular}

Fuente: Eurobarometro 77.4 (año 2012).

Las dificultades económicas se espera que sea el motivo más importante para no realizar viaje turístico y están asociadas con la estratificación social medida a través de variables como son el género, la edad, el hábitat, el nivel de estudios, la ocupación. Esta estratificación sigue marcando la segmentación de los consumidores turísticos, no sólo del tipo de turismo que practican, sino también de la práctica o no de viaje turístico (Ying et al., 2016; Andersen and Curtis, 2015). En muchos países de la Unión Europea se vivió una situación de crisis económica que repercute de forma importante en el consumo. Afecta no sólo a los que están desempleados, sino también a personas que trabajan pero con unos salarios bajos que no les permiten realizar vieja turístico (Herman, 2014) y a los que deciden ahorrar por si persisten los problemas en el futuro. En la tabla 2 se puede apreciar cómo las personas que tienen más dificultades económicas para llegar a fin de mes son los de mediana edad, las mujeres, los que viven en las zonas rurales, los que tienen baja formación. Estas diferencias tienen que verse reflejadas en la realización de viaje turístico.

Podemos resumir diciendo que las personas que tienen menos dificultades económicas para realizar viaje turístico son los jóvenes, los hombres, los que viven en las ciudades, los que tienen elevada formación y los que tienen ocupaciones más elevadas (profesionales con elevada cualificación y directivos), así como los que habitan en los países con modelo de bienestar Nórdico.

El tiempo libre, el otro gran factor que influye en la no realización de viaje turístico, es un derecho está recogido en el artículo 24 de la Declaración Universal de los Derechos Humanos (United Nations, 1948), en el artículo 7 del Pacto Internacional de Derechos Económicos, Sociales y Culturales (United Nations, 1966), en el artículo 2 de la Carta Social Europea (Conseil de l'Europe, 1961), en el artículo 31.2 de la Carta de los Derechos Fundamentales de la Unión Europea (Union Européenne, 2000) y en distintos estatutos de trabajadores. Sin embargo, la realidad es que muchas personas no tienen tiempo libre debido a sus obligaciones laborales, de estudio o familiares. 
Tabla 2

PERSONAS QUE TIENEN DIFICULTADES PARA PAGAR LAS FACTURAS A FINAL DE MES LA MAYOR PARTE DE LAS VECES (\%)

\begin{tabular}{|c|c|c|}
\hline Variables & Categorías & $\begin{array}{r}\% \text { de personas con dificultades } \\
\text { para pagar las facturas a fin de mes la } \\
\text { mayor parte de las veces }\end{array}$ \\
\hline \multirow[t]{6}{*}{ Edad } & $15-24$ años & 13.2 \\
\hline & $25-34$ años & 14.2 \\
\hline & $35-44$ años & 15.1 \\
\hline & $45-54$ años & 16.0 \\
\hline & $55-64$ años & 14.9 \\
\hline & 65 años o más & 11.6 \\
\hline \multirow[t]{2}{*}{ Género } & Hombre & 13.2 \\
\hline & Mujer & 15.0 \\
\hline \multirow{3}{*}{$\begin{array}{l}\text { Tipo de } \\
\text { comunidad }\end{array}$} & Rural & 16.1 \\
\hline & Ciudad tamaño medio & 12.4 \\
\hline & Grandes ciudades & 14.4 \\
\hline \multirow{4}{*}{$\begin{array}{l}\text { Educación. } \\
\text { Años a los que } \\
\text { terminó los } \\
\text { estudios }\end{array}$} & 15 o menos & 24.8 \\
\hline & $16-19$ & 15.2 \\
\hline & $20+$ & 7.5 \\
\hline & Todavía estudiando & 10.4 \\
\hline \multicolumn{2}{|l|}{ Total } & 14.1 \\
\hline
\end{tabular}

Fuente: Eurobarometro 82.3.

Varios autores trabajaron sobre la problemática del tiempo libre e la actualidad (Durán, 2007; Mc Phail Fanger, 2006; Alvaro Page, 1996; Ajenjo Cosp y García Román, 2014; Gershuny and Sullivan, 2003; Haller et al., 2012; Gimenez-Nadal and Molina, 2016). La falta de tiempo libre para dedicar al ocio no afecta por igual a todas las personas, sino que depende de sus condiciones sociales de existencia. En su teoría sobre la distribución del tiempo, Becker (1965) explica como el tiempo es un bien escaso que está asociado con la renta. Esto se puede relacionar con los distintos estados y los distintos grupos sociales. En los estados ricos se cubren fácilmente las necesidades básicas y pueden prescindir del tiempo de trabajo en aras de tener más tiempo libre para el ocio. Davis y Moore (1970) explican porqué los grupos sociales mejor ubicados en los estratos sociales pueden prescindir del tiempo en mayor medida que los grupos sociales peor ubicados. Estas teorías son de gran aplicación para entender los motivos de falta de recursos económicos y de tiempo para realizar viaje turístico que marca diferencias entre Estados con diferentes modelos de Bienestar y entre diferentes grupos sociales.

Sobre la desigualdad en la dedicación del tiempo existen varios estudios, considerando importantes para nuestro contexto los que se recogen en Time Use Surveys (Eurostat, 
2004, 2005, 2009). Tablas con datos específicos por países pueden verse en la web del Harmonised European Time Use Survey (http://ec.europa.eu/eurostat/ramon/statmanuals/ files/KS-RA-08-014-EN.pdf). En la falta de tiempo libre debido a motivos laborales o de estudio, el grupo que tiene menos problemas es el de la tercera edad que ya no trabaja ni estudia. El grupo de edad que probablemente tenga mayores problemas es el de los jóvenes, pues además de tener que estudiar, muchos aprovechan las vacaciones para poder trabajar y así contribuir a generar unos recursos económicos, a ello se dedican programas específicos en la Unión Europea para trabajar en vacaciones (European Youth Portal: http://europa.eu/youth/node/60_en), en distintas actividades.

De los datos de estas encuestas del Time Use Surveys (Eurostat 2009) se concluye que en la distribución del tiempo que dedicamos a las distintas tareas como son el cuidado personal, el trabajo, el estudio, las tareas domésticas, el ocio y el viaje turístico, existen grandes diferencias según el género y los países. Los que dedican menos tiempo al ocio son las mujeres frente a los hombres y considerados conjuntamente hombres y mujeres los que viven en los países con modelos de Estado de Bienestar más desarrollados son los que dedican más tiempo al ocio. Sobre los problemas de tiempo libre en las zonas rurales nos basamos en los estudios de García Sanz (2003), según el cual el tiempo libre en las zonas rurales no se puede sujetar a un horario como en las zonas urbanas, motivo por el que se espera que tengan menor tiempo libre que en las ciudades.

Resumiendo, los que tienen menos dificultades de tiempo libre debido a obligaciones de trabajo y estudio son los jubilados, los que viven en las ciudades frente a las zonas rurales, los que tienen elevada formación y puestos ocupacionales más elevados -a las que como indican Davis y Moore (1970) se da tiempo libre para el disfrute como recompensa- y los que viven en países con modelo de bienestar Nórdico. Respecto a las mujeres, si bien tienen menos probabilidad de nombrar el problema del tiempo libre por trabajo debido a que un menor porcentaje tiene trabajo remunerado, tienen más probabilidad de nombrar tiempo libre por obligaciones familiares debido a que son las que se hacen cargo, en mayor medida, de las tareas no remuneradas. Los que viven en los países con modelo de bienestar Nórdico también se espera que tengan menos problemas de tiempo libre tanto por motivos laborales -debido a que el sistema de relaciones laborales establece mejores condiciones-, cómo por motivos de compromisos familiares -debido a que el Estado se hace cargo en mayor medida de los cuidados asistenciales.

Teniendo en cuenta la suma de los dos factores, económicos y de tiempo libre, se espera que los más aventajados para realizar viaje turístico sean los jóvenes, los hombres, los que tienen formación elevada, los que viven en las ciudades, los que ocupan categorías ocupacionales de profesionales y directivos y los que viven en los países con modelos de Estado de Bienestar Nórdico.

A su vez se espera que la crisis que afectó a varios países de la Unión Europa los últimos años influyese de forma significativa, sobre todo en los países del modelo Meridional (ver tabla 1), dejando a muchas personas con problemas económicos y de tiempo libre al tener que estar pendiente de su trabajo o de encontrar trabajo, así como de tener que realizar tareas domésticas. Además, este problema afectará en mayor medida a las personas de mediana edad, a las mujeres, a los que tienen baja formación, a los que tienen categorías profesionales más bajas. 


\subsection{Hipótesis y representación del modelo de relación entre las variables}

Formulamos siete hipótesis relacionadas con la influencia de variable individuales y contextuales en cuatro variables dependientes: a) la no realización de viaje turístico, b) por motivos económicos, c) por falta de tiempo libre debido a razones laborales/de estudio, d) por falta de tiempo libre debido a compromisos familiares. De cada una de las variables independientes se recoge la influencia directa y mediante la variable de percepción de si la crisis está afectando a su viaje.

Hipótesis 1: La edad afecta a la no realización de viaje, siendo las personas más jóvenes las que tienen menor probabilidad de no realizar viaje y de tener problemas económicos, pero son las que tienen más probabilidad de no viajar por falta de tiempo libre al tener que trabajar o estudiar.

Hipótesis 2: El hábitat afecta a la no realización de viaje, siendo las personas de las grandes ciudades las que tienen menor probabilidad que las de las zonas rurales de no viajar, de tener problemas económicos o de tiempo libre en general.

Hipótesis 3.1: El sexo influye en la no realización de viaje siendo los hombres los que tienen menor probabilidad de no viajar, de tener problemas económicos y de falta de tiempo libre por compromisos familiares, pero tienen más problemas de tiempo libre por tener que trabajar.

Hipótesis 3.2: Además de la influencia directa también se da una influencia mediante la percepción de que la crisis afecta a su viaje turístico, siendo los hombres los que tienen menor probabilidad de considerar que la crisis afecta a sus futuros viajes.

Hipótesis 4.1: Las categorías profesionales siguiendo el modelo de Goldthorpe influyen en la no realización de viaje, siendo los profesionales y directivos los que tienen menor probabilidad de no viajar y de tener problemas económicos o de falta de tiempo libre (excepto en el caso del tiempo libre por motivos laborales que tienen menor probabilidad los parados y los jubilados).

Hipótesis 4.2: Además de la influencia directa también se da una influencia mediante la percepción de que la crisis afecta a su viaje turístico, siendo el grupo de profesionales, directivos y técnicos de alto nivel los que tienen menor probabilidad de considerar que la crisis afecta al viaje turístico.

Hipótesis 5.1: La educación afecta a la no realización de viaje, siendo las personas con estudios superiores las que tienen menor probabilidad de no viajar y de aducir motivos económicos o de falta de tiempo libre.

Hipótesis 5.2: Además de la influencia directa también se da una influencia mediante la percepción de que la crisis afecta a su viaje turístico, siendo las personas con estudios superiores las que tienen menor probabilidad

Hipótesis 6.1: Incluso después de tener en cuenta el efecto de todas las otras variables individuales, el contexto en que se vive según el modelo de Estado de Bienestar influye también en la no realización de viaje, siendo los ciudadanos que viven en países con modelo Nórdico los que tienen menor probabilidad de no viajar y a los que menos afectan los motivos económicos o de tiempo libre. 
Hipótesis 6.2: Además de la influencia directa también se da una influencia mediante la percepción de que la crisis afecta a su viaje turístico, siendo los ciudadanos que viven en los países con modelo Nórdico los que menos consideran que la crisis afecta al viaje turístico.

Hipótesis 7: Las personas que consideran que la crisis afectará a su viaje turístico en el futuro son los que tienen mayor probabilidad de no haber realizado viaje y de aducir motivos económicos o de falta de tiempo libre.

En la fig. 1 se peude ver que tenemos cuatro variables dependientes para explicar (no viajar -viajenotros-, por razones financieras -moteconomicos-, razones de tiempo libre por motivos laborales o de estudio -mottltrabest-, razones de tiempo libre por compromisos familiares -mottlfamilia-) por la influencia de la edad (edad) y hábitat (habitat), la percepción subjetiva de que la crisis está afectando al viaje turístico (crisisafecta) y la influencia directa y mediante crisisafecta del sexo (sexo), categoría ocupacional según el modelo de Goldthorpe (goldthorpe), el nivel de educación (educacion) y el modelo de Estado de Bienestar (esping).

\section{Figura 1}

\section{REPRESENTACIÓN DEL MODELO TEÓRICO DE RELACIÓN DE LAS VARIABLES}

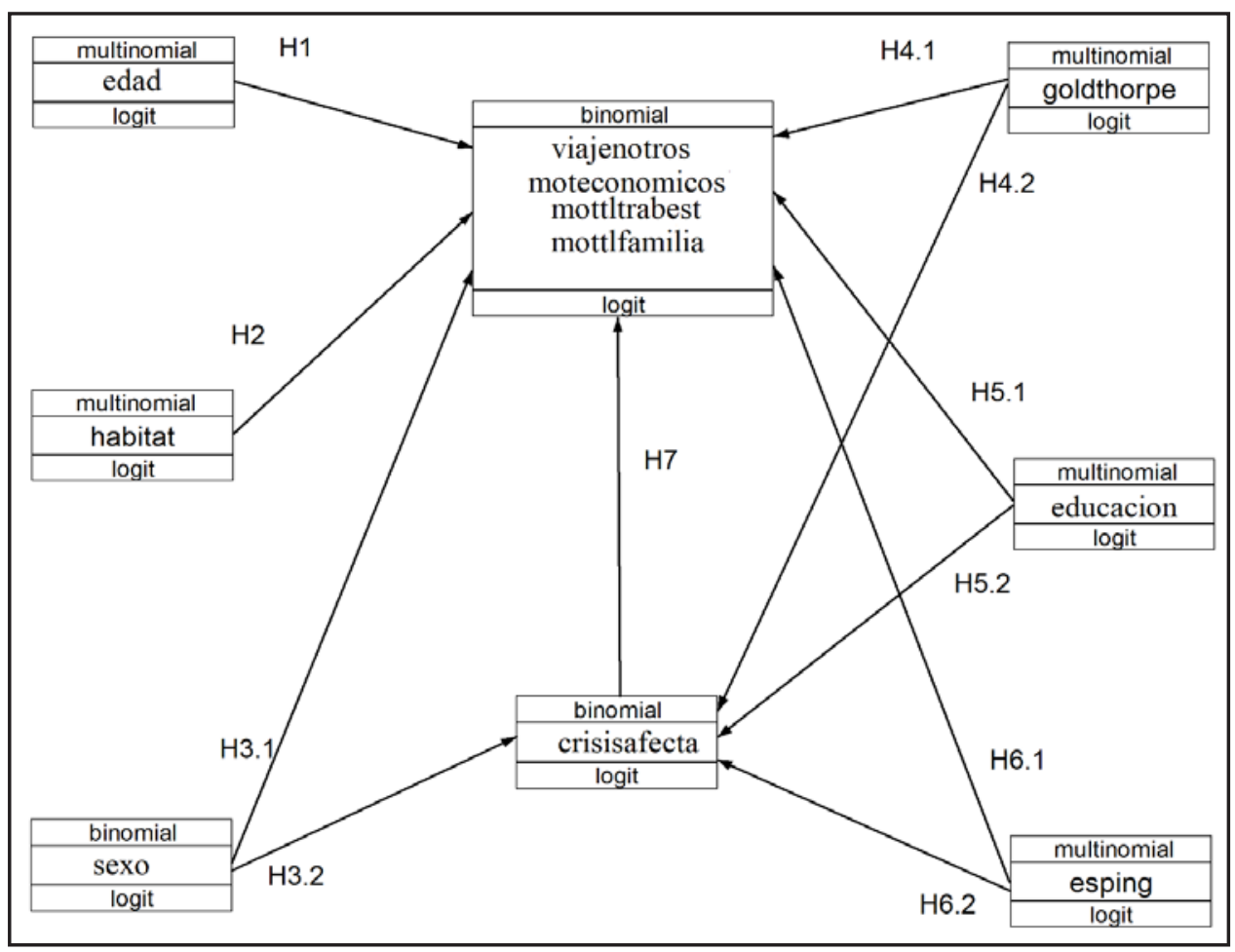




\section{METODOLOGÍA}

Los datos están tomados del flash eurobarometer 414, que hace referencia al turismo realizado en el año 2014. Se consideraron cuatro variables dependientes: realización o no de viaje en el año 2014 (viajenotros), no realizar el viaje por motivos económicos (moteconomicos), por motivos de tiempo libre debido a tener que trabajar o estudiar (mottltrabest), o bien por tener compromisos familiares (mottlfamilia). Todas ellas se trataron como variables binomiales.

Como variables independientes se consideró la edad con tres categorías, jóvenes, adultos y mayores, tomando como categoría base los jóvenes. Hábitat con tres categorías, zonas rurales, ciudades pequeñas y grandes ciudades, tomando como categoría base las grandes ciudades. Sexo con dos categorías, tomando como categoría base el masculino. Categorías profesionales siguiendo el modelo de Goldthorpe (1993, 2007) -con algunos añadidos- diferenciamos entre, profesionales y directivos, empleados no manuales, autónomos, sector primario, técnicos y trabajadores manuales, no cualificados, estudiantes, jubilados, desempleados, tomando como categoría base a os profesionales y directivos. Nivel de educación con tres categorías, básica o menos, media, estudios superiores, tomando como categoría base los estudios superiores. Modelo de Estado de Bienestar siguiendo el modelo de Esping-Andersen $(1990,1999)$-con algunos añadidos- diferenciamos entre Nórdico, Continental, Liberal, Meridional, Europa del Este, tomando como categoría base el modelo Nórdico. La consideración personal de si la crisis va afectar a su viaje en el futuro con dos categorías, tomando como categoría base los que consideran que no afecta.

Para realizar el análisis de la relación entre las condiciones sociales de existencia y la no realización de viaje turístico además de los motivos, después de un análisis bivariado procedimos a realizar un análisis multivariable, analizando la influencia directa de las variables independientes, además de la influencia indirecta de cuatro de ellas con la mediación de cómo la crisis está afectando a la no realización de viaje. Para dicho análisis empleamos las ecuaciones estructurales generalizadas (GSEM).

\section{ANÁLISIS DE LOS DATOS}

\subsection{Análisis descriptivo}

En el total de la UE se realizaron 26805 entrevistas, que oscilaron entre 1505 en Francia y 492 en Malta. Si tenemos en cuenta el total de los entrevistados, no realizó viaje el 28.05\%; pero si lo ponderamos resulta que no realizó viaje el $25.78 \%$ de los ciudadanos de la UE de 15 o más años. Los motivos -podían elegirse varios- son las razones económicas, falta de tiempo libre, de instalaciones para discapacitados, de salud o administrativos. También puede ser debido a que prefieren quedarse en casa. Si analizamos los datos ponderados, el principal motivo es la falta de medios económicos (16.11\% de toda la UE). Le siguen en importancia los de salud (6.72\%), la falta de motivación para viajar y por lo tanto prefieren quedarse en casa $(6.21 \%)$, el tiempo libre debido a compromisos laborales o de estudio (6.01\%), la falta de tiempo libre 
debido a compromisos familiares $(5.27 \%)$, la falta de instalaciones para personas con discapacidades $(0.77 \%)$ y finalmente los problemas administrativos (obtener permiso para visado, etc.).

\section{Tabla 3}

NO REALIZACIÓN DE VIAJE EN EL AÑO 2014 Y MOTIVOS

\begin{tabular}{|l|c|c|c|c|c|c|c|}
\hline \multirow{2}{*}{} & \multirow{2}{*}{ Obs } & \multicolumn{3}{|c|}{ Entrevistados } & \multicolumn{3}{c|}{ Ponderado UE } \\
\cline { 4 - 8 } & & $\%$ & $\begin{array}{c}\text { [95\% Conf. } \\
\text { Interval] }\end{array}$ & $\%$ & \multicolumn{2}{c|}{$\begin{array}{c}\text { [95\% Conf. } \\
\text { Interval] }\end{array}$} \\
\hline $\begin{array}{l}\text { No viajó en el año 2014 pernoctando } \\
\text { al menos una noche fuera de casa }\end{array}$ & 26805 & 28.05 & 27.51 & 28.58 & 25.78 & 25.25 & 26.30 \\
\hline $\begin{array}{l}\text { Motivos de no realizar viaje turístico } \\
\text { (respuesta múltiple) }\end{array}$ & & & & & & & \\
\hline Razones económicas & 26805 & 17.48 & 17.03 & 17.94 & 16.11 & 15.67 & 16.55 \\
\hline $\begin{array}{l}\text { Falta de tiempo libre debido a } \\
\text { compromisos laborales o de estudio }\end{array}$ & 26805 & 4.96 & 4.70 & 5.22 & 6.01 & 5.72 & 6.29 \\
\hline $\begin{array}{l}\text { Falta de tiempo libre debido a } \\
\text { compromisos familiares }\end{array}$ & 26805 & 5.36 & 5.09 & 5.63 & 5.27 & 5.00 & 5.54 \\
\hline $\begin{array}{l}\text { Prefiere quedarse en casa, no está } \\
\text { motivado/a para viajar }\end{array}$ & 26805 & 7.20 & 6.89 & 7.51 & 6.21 & 5.92 & 6.50 \\
\hline $\begin{array}{l}\text { Falta de instalaciones para personas } \\
\text { con discapacidades }\end{array}$ & 26805 & 0.89 & 0.78 & 1.00 & 0.77 & 0.67 & 0.88 \\
\hline Motivos de salud & 26805 & 8.68 & 8.35 & 9.02 & 6.72 & 6.42 & 7.02 \\
\hline $\begin{array}{l}\text { Problemas administrativos (obtener } \\
\text { un visado, etc.) }\end{array}$ & 26805 & 0.20 & 0.14 & 0.25 & 0.18 & 0.13 & 0.23 \\
\hline
\end{tabular}

En esta investigación nos centraremos en analizar como variables dependientes la no realización de viaje y los motivos económicos y de falta de tiempo libre debido a razones laborales o de estudio, y por compromisos familiares. Si nos centramos en los datos ponderados y su intervalo, con un $95 \%$ Conf. Interval podemos ver que existen diferencia significativas de peso entre los tres motivos, siendo el más importante las razones económicas, pero existiendo también diferencia significativa entre los motivos de falta de tiempo libre, es superior el de los compromisos laborales o de estudio que el de compromisos familiares, dado que el valor más alto de compromisos familiares $(5.54 \%)$ es inferior al valor más bajo de compromisos laborales o de estudio (5.72\%).

\subsection{Análisis bivariado}

Para la significación del análisis bivariado se aplica el test de chi cuadrado. Los resultados son que todas las variables dependientes (realización o no de viaje y los motivos de 


\section{Tabla 4}

NO REALIZACIÓN DE VIAJE Y MOTIVOS, SEGÚN LAS CONDICIONES SOCIALES DE EXISTENCIA, MODELO DE ESTADO DE BIENESTAR Y PERCEPCIÓN DE CÓMO AFECTA LA CRISIS

\begin{tabular}{|c|c|c|c|c|c|}
\hline & & & Motiv & s de no & viajar \\
\hline & & 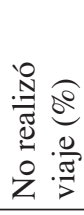 & 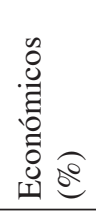 & 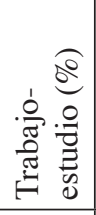 & 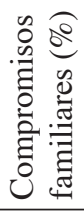 \\
\hline \multirow[b]{3}{*}{ Hábitat } & Rural & 34.1 & 21.9 & 7.3 & 7.0 \\
\hline & $\begin{array}{l}\text { Pequeñas y medianas } \\
\text { ciudades }\end{array}$ & 28.5 & 17.8 & 5.1 & 5.3 \\
\hline & Grandes ciudades & 25.1 & 16.0 & 4.4 & 4.8 \\
\hline \multirow[b]{2}{*}{ Género } & Hombre & 26.8 & 16.4 & 7.3 & 4.7 \\
\hline & Mujer & 31.2 & 20.2 & 4.2 & 6.4 \\
\hline \multirow[b]{3}{*}{ Edad } & 15-34 años & 20.5 & 13.9 & 9.3 & \\
\hline & 35-64 años & 24.9 & 18.4 & 5.5 & \\
\hline & 65 años y más & 38.7 & 17.9 & 1.3 & \\
\hline \multirow[b]{4}{*}{ Nivel de estudios } & Bajo & 51.8 & 31.8 & 5.0 & 7.9 \\
\hline & Medio & 33.0 & 22.4 & 6.5 & 6.7 \\
\hline & Alto & 19.0 & 11.4 & 4.0 & 4.2 \\
\hline & Estudiando & 21.5 & 11.3 & 12.9 & 3.9 \\
\hline \multirow{9}{*}{$\begin{array}{l}\text { Clase social Golthorpe basado en } \\
\text { categorías profesionales }\end{array}$} & Profesionales y directivos & 11.3 & 7.0 & 5.4 & 3.5 \\
\hline & Empleados no manuales & 21.0 & 15.3 & 6.9 & 4.9 \\
\hline & Autónomos & 23.6 & 16.0 & 11.1 & 5.7 \\
\hline & Sector primario & 43.7 & 24.2 & 24.2 & 10.6 \\
\hline & Técnicos y manuales & 30.8 & 21.9 & 11.8 & 6.9 \\
\hline & No cualificados & 43.6 & 29.9 & 5.4 & 12.1 \\
\hline & Estudiantes & 21.7 & 11.1 & 12.8 & 3.6 \\
\hline & Jubilados & 38.3 & 20.2 & 1.2 & 5.2 \\
\hline & Desempleados & 40.7 & 36.1 & 4.5 & 7.1 \\
\hline \multirow{2}{*}{$\begin{array}{l}\text { La crisis afecta a sus vacaciones } \\
\text { de } 2015\end{array}$} & No afecta & 18.3 & 6.0 & 4.3 & 4.0 \\
\hline & Afecta & 37.1 & 27.4 & 6.4 & 6.9 \\
\hline \multirow[b]{5}{*}{ Modelo Estado Bienestar } & ExUS & 33.0 & 24.3 & 6.6 & 7.1 \\
\hline & Meridional & 34.4 & 24.4 & 5.8 & 6.2 \\
\hline & Liberal & 20.3 & 10.2 & 3.6 & 4.0 \\
\hline & Continental & 22.9 & 9.9 & 3.7 & 4.2 \\
\hline & Nórdico & 19.4 & 6.2 & 2.2 & 2.3 \\
\hline $\begin{array}{l}\text { La crisis afecta a sus vacaciones } \\
\text { de } 2015 \text { (\% ciudadanos país) }\end{array}$ & $\begin{array}{l}\text { La crisis afecta: diferencia } \\
\text { porcentaje }\end{array}$ & 4.8 & 9.1 & 4.6 & 5.1 \\
\hline
\end{tabular}


no realización: económicos, falta de tiempo libre por tener que trabajar o estudiar, falta de tiempo libre por compromisos familiares) asocian significativamente con todas las variables independientes (hábitat, género, edad, nivel de estudios, clase social, modelo de estado de bienestar y consideración de que la crisis afecta a sus vacaciones del futuro) al nivel 0.000 excepto para la asociación de la edad con la falta de tiempo libre por compromisos familiares.

Las diferencias entre las distintas categorías de las variables dependientes y las variables independientes pueden verse en la tabla 4. En la no realización de viaje turístico la diferencia entre los que viven en grandes ciudades y los que viven en zonas rurales pasa del $25.1 \%$ al $34.1 \%$. Las mujeres también tienen menos posibilidades de viajar que los hombres. Las personas mayores son las que más sufren el problema $(38,7 \%)$ y los jóvenes los que menos (20.5\%). De los que tienen estudios altos a los que tienen estudios bajos el porcentaje pasa del $19 \%$ al $51.8 \%$. La diferencia entre profesionales y directivos con los trabajadores del sector primario, los no cualificados y los desempleados y enorme, pasando del $11.3 \%$ a cifras de más del $40 \%$. Las personas que viven en países del modelo Nórdico dicen no viajar el $19.4 \%$, pasando a un 33\% para los que viven en países del Este y a un 34.4\% de los países Meridionales. La relación con la percepción subjetiva de la crisis es clara, para aquellos que consideran que les afecta la crisis el porcentaje de los que no viajaron en 2014 es del $37.1 \%$ frente a un $18.3 \%$ de los que no consideran que la crisis les está afectando a su viaje.

Estas variables también afectan a los motivos, siendo en la zona rural mayores los motivos económicos y de tiempo libre en general. Las mujeres muestran mayores motivos económicos y de falta de tiempo libre por obligaciones familiares y menor por trabajo o estudio. Los que tienen estudios bajos tienen mayores motivos económicos y de tiempo libre por obligaciones familiares. El tiempo libre por trabajo afecta más a los que tienen estudios medios. Los jóvenes tienen menos problemas económicos, pero tienen mayor problema de tiempo libre por tener que trabajar o estudiar. Los desempleados son los que tienen mayores dificultades económicas, los del sector primario falta de tiempo libre por trabajo y los no cualificados y labores del hogar tienen falta de tiempo libre por compromisos familiares. Los del modelo de Estado de Bienestar Mediterráneo y la Europa del Este tienen mayores dificultades económicas y de tiempo libre. Los que consideran que la crisis afecta a sus vacaciones tienen mayores dificultades de todo tipo.

\subsection{Análisis multivariable}

Para el ajuste del modelo de las GSEM no son aplicables los ajustes absolutos como en las SEM, sólo son aplicables los relativos Akaike's information criterion (AIC) and Bayesian information criterion (BIC). En aras de buscar un modelo que ajuste bien, se eligieron los modelos con el AIC y el BIC más bajos, realizando la prueba del Likelihood-ratio test (lrtest) para comprobar si son significativas las diferencias. La variable que consideramos si era conveniente incluir o no para comprobar el ajuste es la variable contextual del modelo de Estado de Bienestar (esping). Los resultados (ver tabla 5) fueron positivos, debido a que al dejar de incluir dicha variable en el modelo los AIC y BIC eran significativamente superiores que cuando se incluye dicha variable en el modelo, motivo por el que se incluyó dicha variable. 
Tabla 5

AJUSTE DE LOS MODELOS (AIC, BIC, LRTEST) SEGÚN LA INCLUSIÓN O NO DE LA VARIABLE MODELO DE ESTADO DE BIENESTAR

\begin{tabular}{|c|c|c|c|c|}
\hline Variable dependiente & $\begin{array}{l}\text { Modelo con/ } \\
\text { sin variable } \\
\text { contextual: } \\
\text { modelo de Estado } \\
\text { de Bienestar }\end{array}$ & AIC & $\mathrm{BIC}$ & lrtest \\
\hline \multirow[t]{2}{*}{ No viajó } & Con variable & 61469.5 & 61772.77 & LR $\operatorname{chi} 2=2608.70$ \\
\hline & Sin variable & 64062.2 & 64299.89 & $\begin{array}{l}\text { Prob }>\text { chi } 2= \\
0.0000\end{array}$ \\
\hline \multirow{2}{*}{$\begin{array}{l}\text { No realizó viaje } \\
\text { turístico por dificultades } \\
\text { económicas }\end{array}$} & Con variable & 54237.71 & 54557.37 & LR chi $2=2873.51$ \\
\hline & Sin variable & 57095.23 & 57349.31 & $\begin{array}{l}\text { Prob }>\text { chi } 2= \\
0.0000\end{array}$ \\
\hline \multirow{2}{*}{$\begin{array}{l}\text { No realizó viaje } \\
\text { turístico por falta de } \\
\text { tiempo libre debido a } \\
\text { trabajo o estudio }\end{array}$} & Con variable & 42902.85 & 43222.5 & LR $\operatorname{chi} 2=2326.26$ \\
\hline & Sin variable & 45213.11 & 45467.2 & $\begin{array}{l}\text { Prob }>\text { chi } 2= \\
0.0000\end{array}$ \\
\hline \multirow{2}{*}{$\begin{array}{l}\text { No realizó viaje } \\
\text { turístico por falta de } \\
\text { tiempo libre debido a } \\
\text { compromisos familiares }\end{array}$} & Con variable & 44162.32 & 44465.59 & LR $\operatorname{chi} 2=2354.43$ \\
\hline & Sin variable & 46500.75 & 46738.45 & $\begin{array}{l}\text { Prob }>\text { chi } 2= \\
0.0000\end{array}$ \\
\hline
\end{tabular}

Después de realizar el análisis multivariable GSEM, la variable sexo deja de ser significativa para la no realización de viaje turístico, siendo el $\mathrm{P}>\mathrm{z}$ superior a .05 , motivo por el que no fue incluida en el modelo final. Sin embargo, si nosotros no incluimos en el modelo la variable de categorías profesionales, el sexo pasa a ser significativa. Esto nos viene a decir que la influencia se produce por la diferencia de las mujeres respecto a los hombres en el tipo de profesión. Los resultados para la influencia de las distintas variables sobre la variable dependiente realización o no de viaje turístico (viajenotros) pueden verse en la Figura $2^{1}$. Son los coef. Los resultados del análisis de todas las variables independientes (fila) sobre las dependientes (columna) en OR pueden verse en la tabla 6.

1 No insertamos las figuras de las otras variables dependientes relativas a los distintos motivos (falta de recursos económicos, falta de tiempo libre por tener que trabajar o estudiar, falta de tiempo libre por compromisos familiares) para no incrementar el número de páginas, pero sus resultados OR pueden verse en las tres últimas columnas de la tabla 6. 
Figura 2

NO REALIZACIÓN DE VIAJE TURÍSTICO SEGÚN LAS DISTINTAS VARIABLES INCLUIDAS EN EL MODELO GSEM

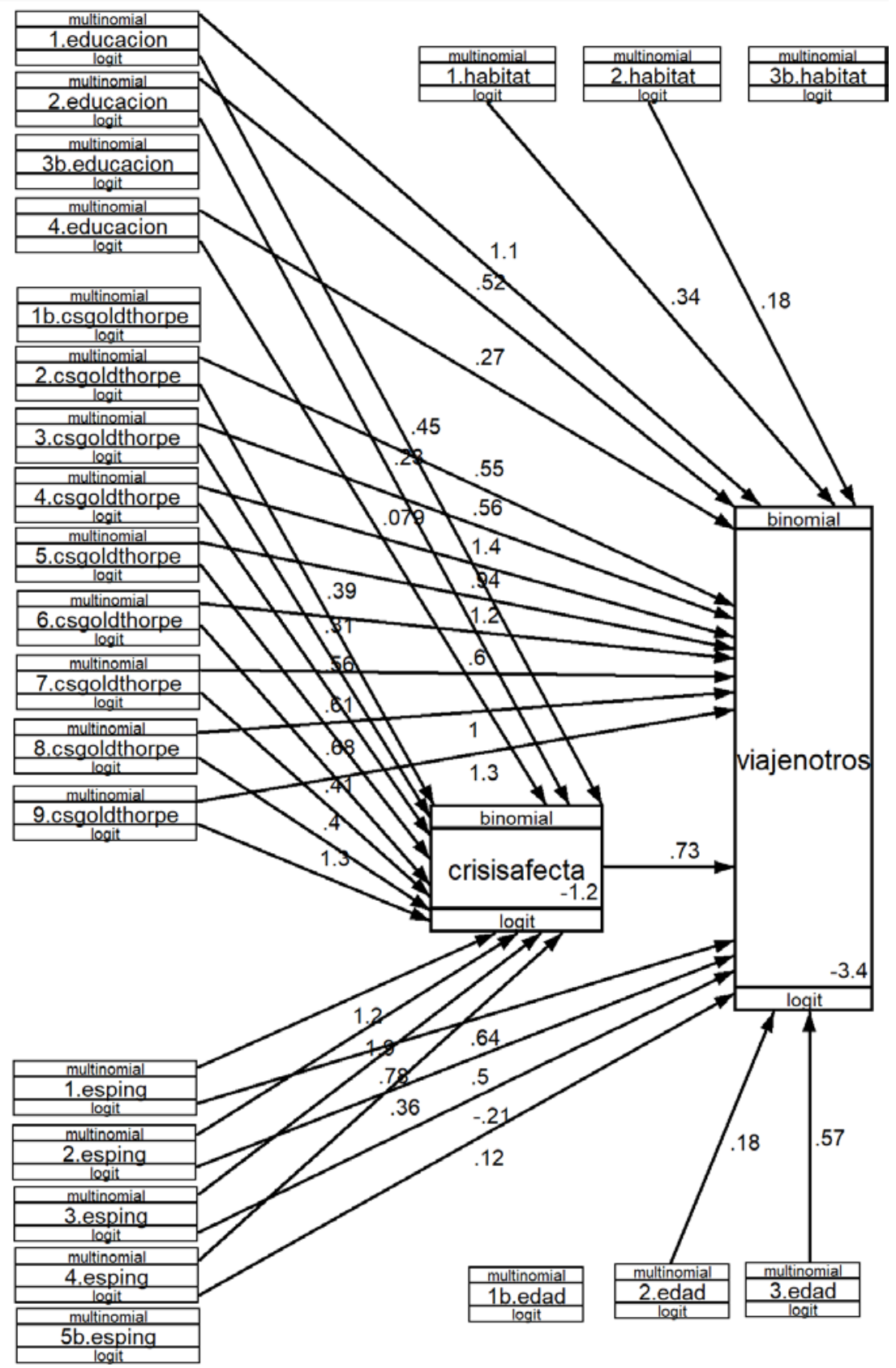

Cuadernos de Turismo, 41, (2018), 17-39 
Tabla 6

RESULTADOS GSEM Y SIGNIFICACIÓN DE LOS COEFICIENTES

\begin{tabular}{|c|c|c|c|c|}
\hline & \multirow[b]{2}{*}{$\begin{array}{l}\text { No viajaron en } \\
\text { el año } 2014\end{array}$} & \multicolumn{3}{|c|}{ Motivos de no realizar viaje turístico } \\
\hline & & Económicos & $\begin{array}{l}\text { Tiempo libre } \\
\text { por tener } \\
\text { que trabajar } \\
\text { o estudiar }\end{array}$ & $\begin{array}{l}\text { Tiempo } \\
\text { libre por } \\
\text { compromisos } \\
\text { familiares }\end{array}$ \\
\hline & OR & OR & OR & OR \\
\hline \multicolumn{5}{|l|}{ Habitat } \\
\hline Rural & $1.41 * * *$ & $1.36^{* * *}$ & $1.75 * * *$ & $1.41 * * *$ \\
\hline Pequeñas y medianas ciudades & $1.19 * * *$ & $1.15 * *$ & $1.37 * * *$ & $1.19 *$ \\
\hline Grandes ciudades & 1.00 & 1.00 & 1.00 & 1.00 \\
\hline Sexo & & $1.07 *$ & $0.67 * * *$ & $1.21 * * *$ \\
\hline \multicolumn{5}{|l|}{ Edad } \\
\hline Jóvenes & 1.00 & 1.00 & 1.00 & \\
\hline Adultos & $1.19 * * *$ & $1.30 * * *$ & $0.70 * * *$ & \\
\hline Mayores & $1.76^{* * *}$ & 1.08 & $0.43 * * *$ & \\
\hline \multicolumn{5}{|l|}{ Educación } \\
\hline Baja & $3.10 * * *$ & $2.68 * * *$ & $1.65^{* * *}$ & $1.51 * * *$ \\
\hline Media & $1.69 * * *$ & $1.72 * * *$ & $1.53 * * *$ & $1.27 * * *$ \\
\hline Alta & 1.00 & 1.00 & 1.00 & 1.00 \\
\hline Estudiando & $1.31 *$ & 1.33 & $1.64 * *$ & 0.88 \\
\hline \multicolumn{5}{|l|}{ CSGoldthorpe } \\
\hline Profesionales y directivos & 1.00 & 1.00 & 1.00 & 1.00 \\
\hline Empleados no manuales & $1.74 * * *$ & $1.93 * * *$ & 1.13 & $1.26^{*}$ \\
\hline Autónomos & $1.75 * * *$ & $1.82 * * *$ & $1.77 * * *$ & 1.36 \\
\hline Sector primario & $3.89 * * *$ & $2.52 * * *$ & $4.05^{* * *}$ & $2.57 * * *$ \\
\hline Técnicos y manuales & $2.55 * * *$ & $2.80 * * *$ & $1.81 * * *$ & $1.91 * * *$ \\
\hline No cualificados y hogar & $3.33 * * *$ & $3.05 * * *$ & 0.86 & $2.64 * * *$ \\
\hline Estudiantes & $1.82 * * *$ & $1.57 * *$ & 1.27 & 0.89 \\
\hline Jubilados & $2.82 * * *$ & $2.71 * * *$ & $0.29 * * *$ & $1.35 * *$ \\
\hline Desempleados y otros & $3.84 * * *$ & $4.86^{* * *}$ & $0.55^{* * *}$ & $1.57 * * *$ \\
\hline
\end{tabular}

Cuadernos de Turismo, 41, (2018), 17-39 


\begin{tabular}{|c|c|c|c|c|}
\hline Crisisafecta & $2.08 * * *$ & $4.28 * * *$ & $1.29 * * *$ & $1.33 * * *$ \\
\hline \multicolumn{5}{|l|}{ Esping-Andersen } \\
\hline Europa Este & $1.90 * * *$ & $3.42 * * *$ & $2.51 * * *$ & $2.89 * * *$ \\
\hline Meridional & $1.66^{* * * *}$ & $2.53 * * *$ & $1.99 * * *$ & $2.17 * * *$ \\
\hline Liberal & $0.81 * *$ & 1.12 & 1.39 & $1.50 *$ \\
\hline Continental & $1.13 * *$ & $1.30 * *$ & $1.44 * * *$ & $1.67 * * *$ \\
\hline Nórdico & 1.00 & 1.00 & 1.00 & 1.00 \\
\hline \multicolumn{5}{|l|}{ Crisisafecta $<-$} \\
\hline Sexo & & $1.37 * * *$ & $1.37 * * *$ & $1.37 * * *$ \\
\hline \multicolumn{5}{|l|}{ Educación } \\
\hline Baja & $1.56 * * *$ & $1.55^{* * *}$ & $1.55^{* * *}$ & $1.55^{* * * *}$ \\
\hline Media & $1.26 * * *$ & $1.27 * * *$ & $1.27 * * *$ & $1.27 * * *$ \\
\hline Alta & 1.00 & 1.00 & 1.00 & 1.00 \\
\hline Estudiando & $1.08^{*}$ & 1.09 & 1.09 & 1.09 \\
\hline \multicolumn{5}{|l|}{ CSGoldthorpe } \\
\hline Profesionales y directivos & 1.00 & 1.00 & 1.00 & 1.00 \\
\hline Empleados no manuales & $1.48 * * *$ & $1.42 * * *$ & $1.42 * * *$ & $1.42 * * *$ \\
\hline Autónomos & $1.36 * * *$ & $1.38 * * *$ & $1.38 * * *$ & $1.38 * * *$ \\
\hline Sector primario & $1.75^{* * *}$ & $1.81 * * *$ & $1.81 * * *$ & $1.81 * * *$ \\
\hline Técnicos y manuales & $1.83 * * *$ & $1.89 * * *$ & $1.89 * * *$ & $1.89 * * *$ \\
\hline No cualificados y hogar & $1.98 * * *$ & $1.76^{* * *}$ & $1.76^{* * *}$ & $1.76^{* * * *}$ \\
\hline Estudiantes & $1.51 * * *$ & $1.48 * * *$ & $1.48^{* * *}$ & $1.48 * * *$ \\
\hline Jubilados & $1.49 * * *$ & $1.43 * * *$ & $1.43 * * *$ & $1.43 * * *$ \\
\hline Desempleados y otros & $3.54 * * *$ & $3.45 * * *$ & $3.45^{* * *}$ & $3.45^{* * *}$ \\
\hline \multicolumn{5}{|l|}{ Esping-Andersen } \\
\hline Europa Este & $3.47 * * *$ & $3.42 * * *$ & $3.42 * * *$ & $3.42 * * *$ \\
\hline Meridional & $6.40 * * *$ & $6.43 * * *$ & $6.43 * * *$ & $6.43 * * *$ \\
\hline Liberal & $2.19 * * *$ & $2.15^{* * *}$ & $2.15 * * *$ & $2.15^{* * *}$ \\
\hline Continental & $1.43 * * *$ & $1.43 * * *$ & $1.43^{* * *}$ & $1.43 * * *$ \\
\hline Nórdico & 1.00 & 1.00 & 1.00 & 1.00 \\
\hline
\end{tabular}

*Coeficientes significativos al nivel 0,05

**Coeficientes significativos al nivel 0,01

$* * *$ Coeficientes significativos al nivel 0,001 
El hábitat influye en la no realización de viaje, en los motivos económicos y de tiempo libre, tanto por razones de trabajo o estudio como por compromisos familiares. Por los OR se puede ver que las personas que viven en las zonas rurales son las que menos viajan y las que manifiestan tener más problemas económicos y de tiempo libre. En las ciudades pequeñas y medianas aunque los problemas son menores que en las zonas rurales, también son mayores que en las grandes ciudades.

Las mujeres tienen más dificultades económicas para viajar que los hombres, tienen más problemas de tiempo libre debido a los compromisos familiares por ser ellas las que suelen realizar los trabajos de la familia no remunerados. Sin embargo, tienen menos dificultades de no viajar por falta de tiempo libre debido a compromisos laborales o de estudio. También son las mujeres las que más perciben que la crisis le va afectar a sus vacaciones en el futuro, teniendo así una influencia directa y otra influencia mediante la repercusión de la crisis.

La edad está influyendo en la no realización de viaje y en los motivos. Los más ancianos son los que manifiestan en mayor medida que no fueron de viaje. Los motivos económicos son manifestados en mayor medida por las personas de mediana edad. Los más jóvenes viajan con pocos medios y las personas mayores tienen ayudas. Son los más jóvenes los que manifiestan con mayor probabilidad para no viajar los motivos de tener que estudiar o trabajar.

El nivel de estudios influye en no viajar y en los distintos motivos. Los que tienen estudios bajos y medios viajaron menos y lo hicieron tanto por motivos económicos como de falta de tiempo libre. Además consideran en mayor medida que la crisis les afectará a sus vacaciones en el futuro. Los que todavía están estudiando viajan menos por falta de tiempo al tener que estudiar o trabajar.

Los profesionales y directivos son los que más viajan y manifiestan en menor medida estar afectados por problemas económicos o de tiempo libre por compromisos familiares. Sin embargo, en el tiempo libre por tener que trabajar o estudiar los que manifiestan el problema en menor medida son los desempleados y los jubilados. Además los profesionales y directivos son los que consideran en menor medida que la crisis les afectará a sus vacaciones del futuro.

La percepción subjetiva de que la crisis afectará a sus vacaciones del futuro influye de forma muy importante, tanto en no realizar viaje turístico como en los distintos motivos. Entre los que consideran que la crisis afecta, frente a los que consideran que no afecta, el OR de no realizar viaje es de 2.08, el OR de la manifestación de motivos económicos es de 4.28, el OR de motivos de falta de tiempo libre por tener que trabajar o estudiar es de 1.29 y por tener compromisos familiares es de 1.33 .

Además de la influencia de las variables individuales consideradas, el contexto donde se vive según el modelo del Estado de Bienestar influye de forma muy importante tanto en la realización o no de viaje como en los motivos de índole financiera o de tiempo libre. Además de la influencia directa también existe una influencia indirecta a través de la consideración de cómo se cree que afectará la crisis en el futuro. Tomando como referencia el modelo Nórdico, los que viven en el modelo Mediterráneo tienen un OR de 1.66. Pero si calculamos el OR total con la mediación de cómo se considera que influirá la crisis el OR se incrementa. En resumen, los que tienen mayor probabilidad de no 
viajar midiendo sólo la influencia directa son los que viven en los países de la Europa del Este, incluso mayor que los que viven en países del Mediterráneo, pero al sumarle la influencia mediada por la consideración de que la crisis afectará a sus viajes en el futuro, la mayor probabilidad de no viajar se da en los países del Meridional, incluso superior a los de la Europa del Este y lo mismo ocurre con la manifestación de motivos económicos o de tiempo libre por tener que trabajar-estudiar o por compromisos familiares. En un punto intermedio se queda el modelo Continental, entre el Meridional y de la Europa del Este respecto al modelo Nórdico. El modelo Liberal en la influencia directa es el que más se parece al modelo Nórdico, teniendo incluso para la no realización de viaje un OR más bajo que en el Nórdico. Pero en la influencia medida por el efecto de la crisis, el modelo Liberal influye todavía más la crisis que en el modelo Continental y por supuesto más que el Nórdico.

\section{CONCLUSIONES}

Después de analizar los resultados se confirman las hipótesis, tanto las que hacen referencia a las variables individuales, como la que hace referencia a la variable contextual del modelo de Estado de Bienestar del país donde se reside. Frente a las consideraciones de que se camina hacia una convergencia entre los Estados de la Unión Europea (Schmitt, 2011; Paetzold, 2013), en lo que respecta al viaje turístico son significativas las divergencias en base a los Modelos de Estado de Bienestar, como se infiere de que al incluir en el modelo dicha variable contextual, el modelo ajusta significativamente mejor.

La primera conclusión es por lo tanto que sigue existiendo una divergencia en el acceso al turismo y en los problemas que llevan a no viajar por motivos económicos o de tiempo libre, bien por tener que trabajar o estudiar, y por compromisos familiares. Por lo tanto, sigue funcionando todavía la teoría de los modelos de Estado de Bienestar, pero lo que es más novedoso de este estudio, es que si bien en principio el modelo Meridional es menos bondadoso que los modelos Continental, Liberal y Nórdico, pero más que el modelo del Este, en la actualidad con la influencia mediadora de la crisis que afectó en mayor medida a los países del Meridional, el acceso al viaje es inferior que en todos los otros países, incluidos los del Este (pasamos de un 33\% en los países del Este a un 34,4\% en los Meridionales).

La otra conclusión hace referencia a que en la no realización de viaje y en los motivos influyen las variables de sexo, edad, formación, hábitat y ocupación, pero con una salvedad que en principio no esperábamos en las hipótesis y es que el sexo si bien influye a nivel bivariado, deja de tener influencia cuando lo consideramos a nivel multivariable en la no realización de viaje, aunque sigue afectando a los motivos, siendo superiores en las mujeres los económicos y de falta de tiempo libre por compromisos familiares y en los hombres la falta de tiempo libre por tener que trabajar. Sin embargo, deja de tener influencia en la no realización de viaje, pues al incluir las variables ocupación y consideración de que la crisis afectará a sus viajes en el futuro, pierde fuerza la variable sexo.

En estas conclusiones también hay que destacar la gran importancia de la consideración subjetiva de que la crisis afectará a los viajes en el futuro, la percepción subjetiva del riesgo (Beck, 1998) que influye como variable mediadora para las variables educación, ocupación 
y modelo de Estado de Bienestar. Por lo tanto, si desapareciese la crisis, se tendería hacia una mayor igualdad en la realización de viaje y se aminoraría el peso de los motivos que llevan a las personas a no realizar viaje como son el financiero y la falta de tiempo libre.

Frente a las teorías de la hipermodernidad como las de Lipovetsky $(2006,2007)$ y la igualdad en el consumo con la desaparición de las diferencias de clase y la globalización con la desaparición de las diferencias de Estados, es así necesario plantear una teoría crítica (Tribe, 2010; Cohen y Cohen, 2012) que reconozca que siguen existiendo diferencias de consumo según los distintos grupos sociales (Bourdieu, 1979). Hay colectivos que tienen dificultades económicas y de tiempo libre para viajar y que además se ven afectadas por las crisis económicas (Semeeding \& Thompson, 2011) de modo heterogéneo entre las distintas zonas de Europa según cómo se vieron afectadas por la crisis. Se produce así una repercusión heterogénea de la crisis en el comportamiento de la población ante el turismo (Eugenio-Martin y Campos-Soria, 2014).

La Unión Europea que reconoció junto con la Organización Mundial del Turismo y la Organización Internacional del Trabajo el derecho de todos los ciudadanos a realizar viaje turístico. De los datos recogidos en los eurobarómetros y a la luz del presente análisis se pone de manifiesto la existencia de grupos desfavorecidos para realizar viaje turístico. La propia Unión Europea manifiesta que es necesario la negociación entre los organismos públicos y privados para facilitar el turismo. Pero ha de reconocer que las políticas no pueden estar encaminadas sólo a gestionar viajes de turismo social, sino que desde una perspectiva crítica es necesario proponer también políticas encaminadas a solucionar los problemas económicos y de tiempo libre para que las personas puedan viajar y aminorar los efectos que produjo la crisis con grandes diferencias entre las zonas de Europa, siendo la más castigada la Mediterránea.

\section{BIBLIOGRAFÍA}

AJENJO COSP, M. y GARCÍA ROMÁN, J. (2014): “Cambios en el uso del tiempo de las parejas. ¿Estamos en el camino hacia una mayor igualdad?”, Revista Internacional de Sociología, 72 (2), pp. 453-476.

ALVARO PAGE, M. (1996): "Diferencias, en el uso del tiempo, entre varones y mujeres y otros grupos sociales", Revista Española de Investigaciones Sociológicas, 74, pp. 291-326.

ANDERSEN, R. y CURTIS, J. (2015): "Social Class, Economic Inequality, and the Convergence of Policy Preferences: Evidence from 24 Modern Democracies", Canadian Review of Sociology, 52 (3), pp. 266-288.

ATELJEVIC, I., HARRIS, C., WILSON, E. y COLLINS, F. L. (2005): "Getting 'entangled': Reflexivity and the 'critical turn' in tourism studies", Tourism Recreation Research, 30, pp. 9-21.

BECK, U. (1998): World Risk Society. Cambridge, Polity Press.

BECKER, G.S. (1965): “A Theory of the allocation of time”. Economic Journal, 75(299), pp. 493-517.

BERTONCELO, E.R.E. (2103): “Classes e prácticas sociais”, Revista Brasileira de Ciências Sociais, 28 (81), pp. 185-2011. 
BOURDIEU, P. (1979): La distinction. Critique sociale du jugement. Paris, Éditions de Minuit.

BRÄNDLE SEÑÁN, G. (2010): “El consumo en tiempos de crisis: una aproximación sociológica a la distribución del gasto en España”. Aposta, 45, pp. 1-24.

CASEY, M.E. (2010): "Low cost air travel: Welcome aboard?", Tourist Studies, 10 (2), pp. 175-191.

CHAN, T. W. y GOLDTHORPE, J. H. (2007): "Social stratification and cultural consumption: The visual arts in England", Poetics, 35, pp. 168-190.

COHEN, E. y COHEN, S.A. (2012): "Current sociological theories and issues in tourism", Annals of Tourism Research, 39 (4), pp. 2177-2202.

COMITE ÉCONOMIQUE ET SOCIAL EUROPEEN (CESE) (2006): Avis sur le Tourisme social en Europe. Disponible en http://eur-lex.europa.eu/legal-content/FR/TXT/PDF/? uri=CELEX:52006IE1155\&from=FR

CONSEIL DE L'EUROPE (1961): Charte Sociale Européenne. Disponible en http://www. cncdh.fr/sites/default/files/charte_sociale_europeenne_revisee_0.pdf

DAVIS, K. y MOORE W.E. (1970): “Some principles of stratification”, American Sociological Review, 10 (2), pp. 242-249.

DEACON, B. (2000): "Eastern European welfare states: the impact of the politics of globalization", Journal of European Social Policy, 10 (2), pp. 146-161.

DOMINGO, M.G., y MORALES, E.S. (2016): "Escenarios desiguales de una Europa en crisis. Aproximación a los efectos de la crisis socioeconómica en los Estados de Bienestar europeos: el caso de Alemania, Suecia y España”, Acta Sociológica, 69, pp. 181-205.

DURÁN, M.A. (2007): El valor del tiempo. Madrid, Espasa.

ESPING-ANDERSEN, G. (1990): Three Worlds of Welfare Capitalism. Cambridge, Polity Press.

ESPING-ANDERSEN, G. (1999): Social Foundations of Postindustrial Economies. Oxford, Oxford University Press.

ESPING-ANDERSEN, G. (2000a): “Towards the Good Society, Once Again?”, en EspingAndersen, G. (Ed.) Why we need a New Welfare State. Oxford, Oxford University Press, pp. 1-25.

ESPING-ANDERSEN, G. (2000b): “A New Gender Contract”, en Esping-Andersen, G. (Ed.) Why we need a New Welfare State. Oxford, Oxford University Press, pp. 68-95.

EUGENIO-MARTIN, J.L., y CAMPOS-SORIA, J.A. (2014): "Economic crisis and tourism expenditure cutback decision", Annals of tourism Research, 44, pp. 53-73.

EUROPEAN YOUTH PORTAL: Holiday work. Disponible en http://europa.eu/youth/ node/60_en

EUROSTAT (2004): How Europeans spend their time - Everyday life of women and men. Disponible en http://www.unece.org/fileadmin/DAM/stats/gender/publications/MultiCountry/EUROSTAT/HowEuropeansSpendTheirTime.pdf

EUROSTAT (2005): Comparable time use statistics - National tables from 10 European countries, Working paper. Disponible en http://ec.europa.eu/eurostat/ documents/3888793/5833013/KS-CC-05-001-EN.PDF/5af70d49-9012-444d-b6a0f28a7677d8e4 
EUROSTAT (2009): Harmonised European time use surveys. 2008 guidelines. Disponible en http://ec.europa.eu/eurostat/ramon/statmanuals/files/KS-RA-08-014-EN.pdf

FLAQUER, L. (2004): "La articulación entre familia y Estados de bienestar en los países de la Europa del sur”, Papers: Revista de Sociología, 73, pp. 25-78.

GARCÍA SANZ, B. (2003): Sociedad y desarrollo rural. Madrid, Ministerio de Agricultura, Pesca y Alimentación.

GERSHUNY, J. y SUllivAN, O. (2003): “Time Use, Gender, and Public Policy Regimes", Social Politics, 10 (2), pp. 205-228.

GIMENEZ-NADAL, J.I. y MOLINA, J.A. (2016): "Commuting time and Household Responsibilities: Evidence Using Propensity Score Matching”, Journal of Regional Science, 56 (2), pp. 332-359.

GOLDTHORPE, J.H. (2013): "Back to Class and Status: Or Why a Sociological View of Social Inequality Should Be Reasserted”, Revista Española de Investigaciones Sociológicas, 137, pp. 43-58.

HALL, D. y BRONW, F. (2012): “The Welfare Society and Tourism: European Perspectives", en Scott McCabe, Lynn Minnaert y Anya Diekmann, Social Tourism in Europe. Theory and Practice. Bristol: Channel View Publications, pp. 108-121.

HALLER, M., HADLER, M. y KAUP, G. (2012): "Leisure Time in Modern Societies: A new Source of Boredom and Stress?", Social Indicators Research, 111, pp. 403-434.

HEMERIJCK, A. (2000): "The Self-Transformation of the European Social Model(s)", en Esping-Andersen, G. (Ed.) Why we need a New Welfare State. Oxford, Oxford University Press, pp. 173-214.

HERMAN, E. (2014): "Working Poverty in the European Union and its Main Determinants: an Empirical Analysis", Engineering Economics, 25 (4), pp. 427-436.

KOUBA, L. y GROCHOVÁ, L. (2013): "The European Welfare State from the Prospect of new EU Member States”, Acta Universitatis Agriculturae et Silviculturae Mendelianae Brunensis, LXI (7), pp. 2327-2335.

LIPOVETSKY,G.(2006): Le Bonheur paradoxal: essai sur la sociétéd'hyperconsommation. Paris, Gallimard.

LIPOVETSKY, G. (2004): Les Temps hypermodernes. Paris, Grasset.

MC PAIL FANGER, E. (2006): Voy atropellando tiempos: Género y tiempo libre. México DF, UAM-X.

McCABE, S. (2009): "Who needs a holiday? Evaluating social tourism", Annals of Tourism Research, 36 (4), pp. 667-688.

MINNAERT, L., MAITLAND, R., y MILLER, G. (2009): “Tourism and social policy: The value of social tourism", Annals of Tourism Research, 36 (2), pp. 316-334.

MORENO, L. (2001): “La 'vía media' española del modelo de bienestar mediterráneo", Papers: Revista de Sociología, 63/64, pp. 67-82.

ORGANISATION INTERNATIONAL DU TOURISME SOCIALE (OITS) (2018): What is Social Tourism?. Disponible en http://www.oits-isto.org/oits/public/section.jsf?id=39

PAETZOLD, J. (2013): “The Convergence of Welfare State Indicators in Europe", European Journal of Social Security, 15 (1), pp. 28-54.

PRITCHARD, A., MORGAN, N. y ATELJEVIC, I. (2011): "Hopeful tourism: A new transformative perspective", Annals of Tourism Research, 38 (3), pp. 941-963. 
SAPIR, A. (2006): "Globalization and the Reform of European Social Models", Journal of Common Market Studies, 44 (2), pp. 369-390.

SCHMITT, C. (2011): "Explaining convergence of OECD welfare states: a conditional approach", Journal of European Social Policy, 21 (2), pp. 120-135.

SMEEDING, T. y J. THOMPSON (2011): "Recent trends in income inequality", Research in Labour Economics, 32, pp. 1-50.

TRIBE, J. (2010): "Tribes, territories and networks in the tourism academy", Annals of Tourism Research, 37 (1), pp. 7-33.

UNION EUROPÉENNE (2000): Charte des droits fondamentaux de l'Union Européenne. Disponible en http://www.europarl.europa.eu/charter/pdf/text_fr.pdf

UNITED NATIONS (UN) (1948): Universal Declaration of Human Rights. Disponible en http://www.refworld.org/docid/3ae6b3712c.html

UNITED NATIONS (UN) (1966): International Covenant on Economic, Social and Cultural Rights. Disponible en http://www.refworld.org/docid/3ae6b36c0.html

UNITED NATIONS (UN) (2001): Global Code of Ethics for Tourisms. Disponible en http://dtxtq4w60xqpw.cloudfront.net/sites/all/files/docpdf/gcetbrochureglobalcodeen. pdf

WARNIER, J.P. (1999): La mondialisation de la culture. Paris, La Découverte.

WUGGENIG, U. (2007): "Comments on Chan and Goldthorpe: Pitfalls in testing Bourdieu's homology assumptions using mainstream social science methodology Social stratification and cultural consumption: The visual arts in England", Poetics, 35, pp. 306-316.

YING, T., NORMAN, W. y ZHOU, L. (2016): "Is social class still working? Revisiting the social class division in tourist consumption", Current Issues in Tourism, 19 (14), pp. $1405-1424$. 
